\title{
Burden of Breast Cancer in the Arab World: Findings from Global Burden of Disease, 2016
}

\author{
M. Jawad Hashim¹, Fatima A. Al-Shamsi ${ }^{1}$, Noura A. Al-Marzooqi' ${ }^{1}$, Sarah S. Al-Qasemi' ${ }^{1}$, Ali H. Mokdad ${ }^{2}$, Gulfaraz Khan ${ }^{3, *}$ \\ ${ }^{1}$ Department of Family Medicine, College of Medicine and Health Sciences, United Arab Emirates University, Al Ain, United Arab Emirates \\ ${ }^{2}$ Institute of Health Metrics and Evaluation, University of Washington, Seattle, Washington \\ ${ }^{3}$ Department of Microbiology and Immunology, College of Medicine and Health Sciences, United Arab Emirates University, Al Ain, United Arab Emirates
}

\author{
ARTICLE INFO \\ Article History \\ Received 10 May 2018 \\ Accepted 6 September 2018 \\ Keywords \\ Breast cancer \\ Arab region \\ cancer epidemiology \\ sociodemographic index

\section{Abbreviations} \\ SDI, sociodemographic index \\ YLDs, years lived with disability \\ YLL, years of life lost \\ DALYs, disability adjusted life years \\ GBD, global burden of disease
}

\begin{abstract}
Epidemiology of breast cancer in the Arab region is understudied as compared with Western countries. We aimed to examine breast cancer epidemiology in Arab countries from 1990 to 2016. We analyzed the Global Burden of Disease, 2016 data for breast cancer among women in 22 Arab countries. Epidemiological measures including incidence, mortality, and disability adjusted life years (DALYs) were analyzed for breast cancer in women from 1990 to 2016. We also measured the burden of breast cancer stratified by the sociodemographic index (SDI). Our analysis indicates that the incidence of breast cancer in Arab women has risen over the past 26 years, but is still lower than global averages. In 2016, there were 45,980 new cases $(28 / 100,000)$ and 20,063 deaths $(11 / 100,000)$ in the region. The burden of breast cancer as estimated by DALYs was also lower than the global rates and tended to increase with increasing SDI. Although some studies have reported that Arab women present with breast cancer at a younger age, our analysis of age-specific rates, indicates that this is not statistically significant. Our findings indicate that a comprehensive plan to improve public awareness, screening, diagnosis, and treatment is required to reduce the growing burden of breast cancer in the Arab world.
\end{abstract}

(c) 2018 Atlantis Press International B.V. This is an open access article under the CC BY-NC license (http://creativecommons.org/licenses/by-nc/4.0/).

\section{INTRODUCTION}

Despite major efforts at early detection and new treatments, breast cancer claims the lives of over 520,000 women every year [1]. Risk factors for breast cancer include older age especially postmenopausal status, family history of breast cancer, earlier age of menarche, delayed first pregnancy, lower parity, and shorter duration of breastfeeding $[2,3]$. In particular, concerns have been raised over the apparent increase in the rates of breast cancer in the Arab region [4,5]. A 2007 literature review estimated that breast cancer accounts for 13-35\% of all female cancers in Arab countries [6]. In the same review, based on the unadjusted data, there was a trend toward earlier age of onset as well as presentation at advanced stages among Arab women [6]. Notably, the stage of disease was more advanced in Arab women at the time of diagnosis in every age group, indicating perhaps, either late detection or reluctance to seek medical care [7]. Screening programs are almost nonexistent in many Arab countries [6].

Small-scale studies on breast cancer in Arab women have provided a context for a wider perspective on the burden of suffering. These studies have focused on familial risk and the quality of life among women with breast cancer. A few such studies have focused on the quality of life as an indicator of gaps in medical care and the burden of human suffering. A review of 9 similar studies reported

*Corresponding author.Email: g_khan@uaeu.ac.ae a wide variation in the quality of life among Arab women with breast cancer [8]. For instance, about $40 \%$ of women with breast cancer at an oncology center in Egypt reported poor quality of life [8]. The findings indicated that at least some of the variation can be explained by the education and socioeconomic status of these women. Thus, the epidemiology of breast cancer in this region is complex, and multiple risk factors appear to be involved.

The Arab region consists of 22 countries located in the Middle East and North Africa. The region shares ties of language and culture. Yet, it is also fairly diverse in terms of economic development, education, and healthcare infrastructure. Several countries in the region are experiencing healthcare crises due to internal conflicts [9]. The epidemiology of breast cancer in the Arab region has not been studied comprehensively. We sought to analyze the burden of disease due to breast cancer among women in the Arab region using the Global Burden of Disease (GBD) database. In addition, we aimed to examine breast cancer incidence in Arab countries over the past two decades and predict future trends.

\section{MATERIALS AND METHODS}

We analyzed the data from the GBD database compiled by the Institute of Health Metrics and Evaluation at the University of Washington, Seattle (www.healthdata.org). The methodology and 
approach used by the GBD project to collect and analyze the data has been described extensively elsewhere $[10,11]$. The sources of data include nationally representative surveys, cancer registries, vital registration systems, and healthcare usage data. The GBD was selected because of its rigorous methods in adjusting for differences in sources, and the variety of models used to generate estimates based on the complexity of disease, natural history, and regional data. Moreover, advanced statistical modeling techniques are used to generate regional estimates. We used the GBD 2016 data to extract the incidence, mortality, and disability adjusted life years (DALY) of female breast cancer in Arab countries from 1990 to 2016. Future trends in breast cancer incidence in the region (2016-2025) were estimated by extrapolation of prior data using non-linear regression. Statistical data analysis was performed using SPSS version 24 (IBM SPSS Inc., 2017).

\subsection{Countries in the Arab Region}

Based on the membership of the Arab League (2013), 22 countries make up the Arab world. These 22 countries (Algeria, Bahrain, Comoros, Djibouti, Egypt, Iraq, Jordan, Kuwait, Lebanon, Libyan, Mauritania, Morocco, Occupied Palestinian Territory, Oman, Qatar, Saudi Arabia, Somalia, Sudan, Syria, Tunisia, United Arab Emirates, and Yemen) are located in the Middle East and North Africa. We grouped these countries into three categories based on their sociodemographic index (SDI), which measures the average income per person, average years of schooling, and total fertility rate $[12,13]$. GBD classifies countries into five SDI categories: high, high-middle, middle, low-middle, and low. To have sufficient numbers in each category, we merged high with high-middle and low-middle with low SDI countries in this study.

\subsection{Outcome Metrics}

To estimate the burden of breast cancer in the Arab world, a number of outcomes were evaluated, including incidence, mortality, and disability adjusted life years (DALYs). DALYs is the sum of years lived with disability (YLDs) and years of life lost (YLLs) due to premature mortality. Thus, DALYs may be considered a better reflection of the burden of suffering caused by a disease than mortality rates. These measures were also analyzed in the context of social development as reflected by the SDI.

\subsection{Statistical Analysis}

Data were initially downloaded into Microsoft Excel and subsequently analyzed using SPSS statistical software (IBM SPSS, Inc., Chicago, 2017). Nonparametric (related-samples Wilcoxon signed rank) tests were used to compare age groups in breast cancer incidence. A $p$ value of $<0.05$ was considered statistically significant.

\section{RESULTS}

Incidence of breast cancer in the Arab world has risen gradually between 1990 and 2016 (Figure 1). The rate of increase appears to be similar to the global trend. Without any intervention, it is predicted that the incidence is likely to continue to rise over the next

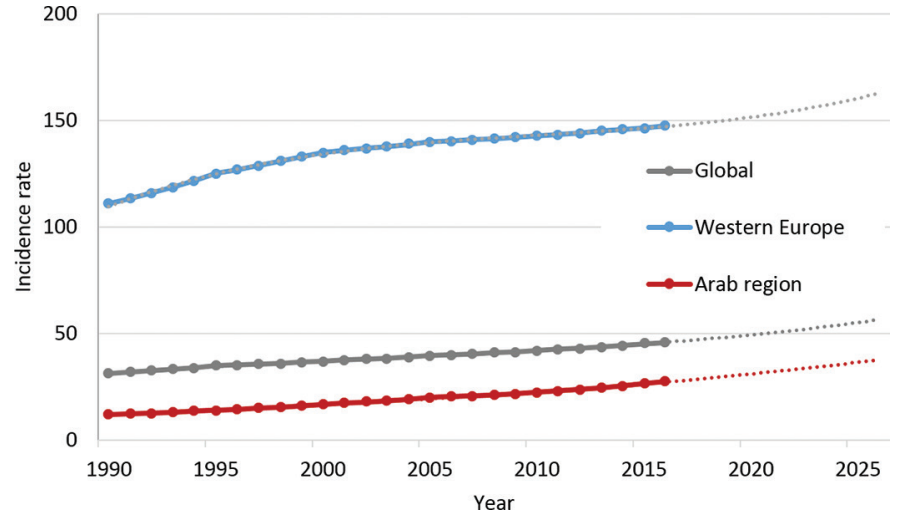

Figure 1 Trends in breast cancer in the Arab world, 1990-2016, and projections to 2025. Notes: Incidence rates of breast cancer among women (all ages) per 100,000 persons. Projection estimates up to 2025 are in dotted lines.

10 years, both globally and in the Arab world (Figure 1). The overall burden of breast cancer in the Arab world is summarized in Table 1. The incidence of breast cancer in 2016 among women in the Arab region $(28 / 100,000)$ was lower than the global mean $(46 / 100,000)$. However, compared with those in Western Europe $(148 / 100,000)$, the incidence rates were strikingly lower. The overall burden of suffering in terms of DALYs was also lower in Arab countries.

More developed Arab countries with higher SDIs, tended to have a higher burden of suffering from breast cancer. This was further corroborated from country-specific data (Table 2). Lebanon had the highest incidence rate among Arab nations, followed by Bahrain and Morocco. In general, the mortality rates also followed a similar trend, with more deaths per 100,000 in high SDI countries. Not surprisingly, countries such as Egypt, Morocco, and Iraq, which have large populations, had the highest total number of deaths. However, the true burden of suffering is reflected by the rate of DALYs (Table 2 and Figure 2).

Analysis of breast cancer incidence by age revealed that the rates among younger Arab women (30-59 year olds) are not significantly different from their global counterparts (Figure 3). By contrast, rates among older Arab women (60 and above) were significantly lower.

\section{DISCUSSION}

Analysis of GBD 2016 data revealed that the incidence of breast cancer in the Arab world is rising. The rate of rise is comparable with the global trend and it is predicted to continue to rise if no interventions are implemented. For many years, controversy has surrounded the issue of the age of onset of breast cancer in Arab women $[4,14]$. Studies have suggested that Arab women present with breast cancer at younger ages $[15,16]$. In contrast to these reports, our analysis using age-specific incidence rates show no evidence of earlier onset.

\subsection{Overall Burden of Disease}

Breast cancer incidence has been rising in Arab countries. This increase in the incidence may be partly due to lifestyle changes being adopted by Arab women, such as late marriage, delayed first 
Table 1 Burden of breast cancer in the Arab world, 2016

\begin{tabular}{lcccr}
\hline & $\begin{array}{c}\text { Incidence } \\
\text { rate }\end{array}$ & $\begin{array}{c}\text { DALY } \\
\text { rate }\end{array}$ & $\begin{array}{c}\text { Mortality } \\
\text { rate }\end{array}$ & $\begin{array}{c}\text { Total } \\
\text { deaths }\end{array}$ \\
\hline High SDI & 39 & 364 & 10 & 2599 \\
Middle SDI & 25 & 359 & 10 & 10,296 \\
Low SDI & 17 & 367 & 10 & 7168 \\
Arab world & 28 & 363 & 11 & 20,063 \\
Western Europe & 148 & 801 & 39 & 85,042 \\
Global & 46 & 405 & 15 & 535,341 \\
\hline
\end{tabular}

All figures are for the year 2016. All the rates are per 100,000; DALY, disability adjusted life years; SDI, socio-demographic index.

Table 2 Country-specific data on breast cancer, 2016

\begin{tabular}{lccrr}
\hline Country & $\begin{array}{c}\text { Incidence } \\
\text { rate }\end{array}$ & $\begin{array}{c}\text { DALY } \\
\text { rate }\end{array}$ & $\begin{array}{c}\text { Death } \\
\text { rate }\end{array}$ & $\begin{array}{c}\text { Total } \\
\text { deaths }\end{array}$ \\
\hline Global & $\mathbf{4 6}$ & $\mathbf{4 0 5}$ & $\mathbf{1 5}$ & $\mathbf{5 3 5 , 3 4 1}$ \\
Algeria & 27 & 411 & 12 & 2392 \\
Arab region & 28 & 363 & 11 & 20,063 \\
Bahrain & 44 & 458 & 13 & 71 \\
Comoros & 12 & 317 & 10 & 37 \\
Djibouti & 10 & 259 & 8 & 40 \\
Egypt & 19 & 237 & 7 & 3231 \\
Iraq & 19 & 468 & 14 & 2623 \\
Jordan & 32 & 427 & 13 & 474 \\
Kuwait & 36 & 276 & 8 & 145 \\
Lebanon & 84 & 561 & 21 & 622 \\
Libya & 37 & 360 & 10 & 320 \\
Mauritania & 12 & 317 & 10 & 205 \\
Morocco & 44 & 642 & 18 & 3094 \\
Oman & 17 & 194 & 6 & 89 \\
Palestine & 14 & 320 & 10 & 250 \\
Qatar & 39 & 403 & 10 & 64 \\
Saudi Arabia & 25 & 201 & 6 & 878 \\
Somalia & 12 & 370 & 11 & 586 \\
Sudan & 18 & 338 & 10 & 1916 \\
Syria & 12 & 165 & 5 & 465 \\
Tunisia & 44 & 553 & 18 & 1014 \\
UAE & 36 & 389 & 10 & 257 \\
Western Europe & 148 & 801 & 39 & 85,042 \\
Yemen & 13 & 323 & 9 & 1290 \\
\hline
\end{tabular}

All figures are for the year 2016. All the rates are per 100,000. Notes: DALY, disability adjusted life years.

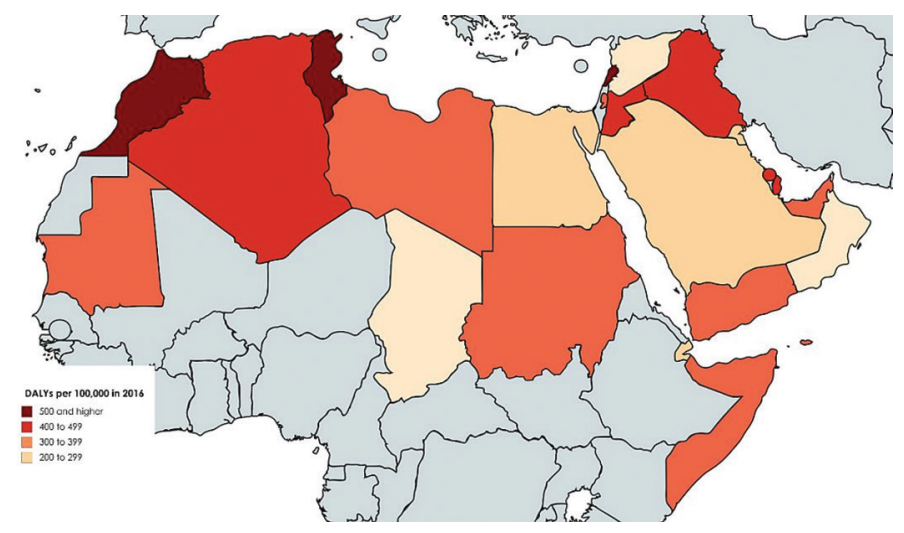

Figure 2 Geographical distribution of breast cancer burden in the Arab world, 2016. Notes: DALY rates per 100,000 persons in 2016. Darker red hues indicate higher rates.

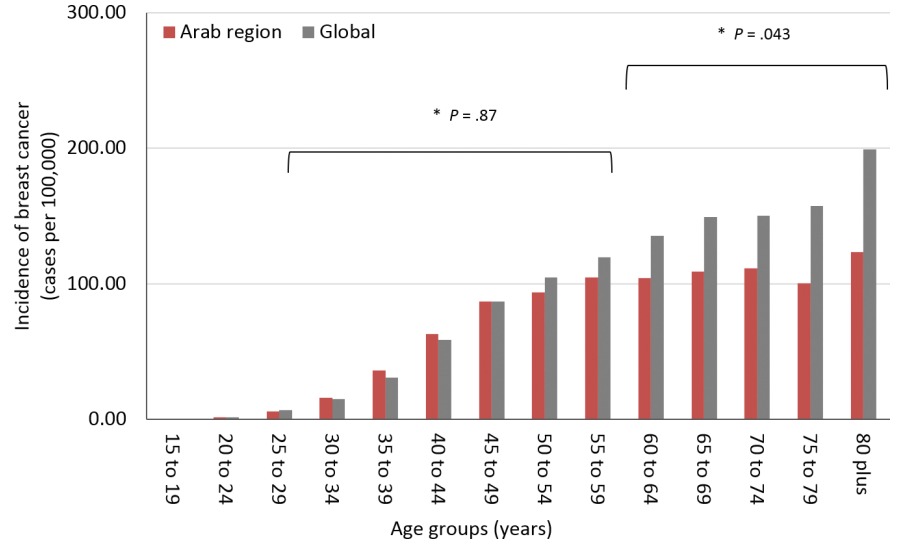

Figure 3 Age distribution of breast cancer incidence in the Arab world, 2016. Incidence figures in the age bracket 30-59 years are not significantly different in Arab women compared with women globally ( $p=0.87$, related-samples Wilcoxon signed rank test), whereas in the age bracket $60-80+$, are higher for women globally $(p=0.043)$.

pregnancy, having fewer children, taking oral contraceptives, lack of physical activity/obesity, and smoking [17-20]. It is also possible that this increase is partly due to improved detection and diagnosis in some Arab countries over the past 26 years. Population growth and aging could also be possible factors contributing to the increased burden [17]. Although the incidence of breast cancer in Arab countries is rising, it is still less than the global average and only one-fifth of that in Western Europe. In a comparative study of Arab and Jewish women with breast cancer in Israel, it was found that the age-adjusted incidence rates were lower among the former [7]. However, some researchers have questioned the validity of this observation, citing the possibility of undetected cases [15]. Others have defended the observation based on data from limited, but fairly reliable population-based cancer registries in Arab countries [14]. Our data also indicate that there is a noticeable difference in the burden of breast cancer between Arab and European countries. Moreover, when Arab countries are grouped on the basis of SDI, taking into account not only the average income per person, but also the average years of schooling and the total fertility rate, a trend is discernible. The incidence rate is higher in high SDI countries as compared with less-affluent, low-SDI countries. What is contributing to the higher incidence rates in high-SDI countries is not clear. It could be due to better reporting in these countries as well as higher prevalence of risk factors such as obesity [21]. Although the incidence is lower in low-SDI countries, the mortality rates are comparable across all SDI groups, implying that women in less-affluent countries have a shorter survival.

\subsection{Age at Diagnosis}

The age of the onset of breast cancer in the Arab world is controversial $[4,15,16]$. Earlier studies have generally relied on the use of mean age or unadjusted age distribution of breast cancer to infer that the age at the onset of breast cancer is lower among Arab women [15]. Najjar and Easson [15] found that the mean age at diagnosis was 48 years (SD 2.8), almost a decade younger compared with that among western women. In a large series of breast cancer cases from Lebanon, the mean age was 49.8 years, with $50 \%$ of the women presenting at ages less than 50 years [16]. By contrast, 
only about $23 \%$ of breast cancer cases are diagnosed in women under the age of 50 in the United States [22]. Similarly, a statistically significant difference was found in the mean age at diagnosis in Arab Palestinian women (51.5 years) compared with that among Jewish women (55.9 years) [23]. However, it should be noted that these comparisons of unadjusted rates can be misleading due to the fact that the Arab region has a much younger population. However, age-specific incidence is considered a more appropriate measure than the mean age at diagnosis, and has important implications for public health policy [14]. Our data, based on age-specific incidence rates, indicate that the onset of breast cancer in Arab women is in fact comparable with global rates. These observations imply that there are no inherent biological differences between Arab and nonArab women in terms of breast cancer onset. By contrast, the incidence rates in older Arab women (60 and above) are significantly lower. Cultural reasons could account for the lower rates of breast cancer reported among these women. Elderly Arab women are less likely to seek breast cancer screening [24].

\subsection{Recommendations and Policy Implications}

The increasing trend in the burden of breast cancer in the Arab world and the complexity of risk factors associated with this malignancy warrants a multipronged approach. Improvements in public awareness, lifestyle practices, early detection and diagnosis, better access to affordable treatment, and more palliative care, are all important issues that need to be addressed. These changes can only be effectively implemented by a joint effort from policy makers, healthcare professionals, and the general public. It has been suggested that breast cancer screening for Arab women should start at an earlier age as compared with guidelines from Western countries [15]. Our findings do not support changing the screening guidelines, but warrant more efforts in improving access and uptake of screening. Practical strategies such as mobile mammography units and family health programs have been shown to improve screening rates in resource-limited settings [25].

\subsection{Limitations}

Although based on the best available data, our study is nevertheless limited by the quality of information reported from the region. Cancer data in this region are often based on hospital registries or retrospective case series. Moreover, social stigma associated with breast cancer can lead to underreporting. Statistical analysis of country-level averages can also be misleading and should be interpreted with caution. Temporal data are susceptible to changes in case definition, reporting mandates and healthcare initiatives such as screening, and improved access to care. Finally, breast cancer in the region should be viewed within a wider perspective of other more pressing healthcare needs of women, such as ischemic heart disease and mental health [26].

\section{CONCLUSION}

Breast cancer data for the 22 Arab countries in the Middle East and North Africa collated by the GBD initiative, suggests a rising trend for more than two decades. The findings from this study endorse an urgent need for the Arab countries to improve screening and diagnosis, as well as treatment and palliative care [17]. Even with these interventions, the predicted trends suggest that the burden of breast cancer will continue to rise. Unfortunately, the Arab region is also facing a rising burden of a number of other noncommunicable diseases and to reduce the burden due to breast cancer is a major challenge [28]. This is particularly the case for low-income Arab countries which have poorly developed healthcare systems. Furthermore, civil conflicts, added onto the already existing social, political, and economic instabilities, inevitably increase the burden of suffering from breast cancer in some of the countries in the region [11].

\section{ACKNOWLEDGMENTS}

We thank the Institute of Health Metrics and Evaluation for providing access to the GDB data.

\section{AUTHORSHIP CONTRIBUTION}

$\mathrm{MJH}$ and GK conceived the idea, analyzed the data, and wrote the first draft of the manuscript. FAA, NAA, and SSA participated in the data preparation. AHM provided critical guidance on the manuscript and data analysis. All authors read the manuscript and provided feedback.

\section{CONFLICTS OF INTEREST}

The authors have no conflicts of interest to declare.

\section{REFERENCES}

[1] Forouzanfar MH, Foreman KJ, Delossantos AM, Lozano R, Lopez AD, Murray CJL, et al. Breast and cervical cancer in 187 countries between 1980 and 2010: a systematic analysis. Lancet 2011;378;1461-84.

[2] Sanderson M, Pérez A, Weriwoh ML, Alexander LR, Peltz G, Agboto V, et al. Perinatal factors and breast cancer risk among Hispanics. J Epidemiol Glob Health 2013;3;89-94.

[3] Morales L, Alvarez-Garriga C, Matta J, Ortiz C, Vergne Y, Vargas $\mathrm{W}$, et al. Factors associated with breast cancer in Puerto Rican women. J Epidemiol Glob Health 2013;3;205-15.

[4] Azim Jr HA. Breast cancer in young Arab women: do ethnic differences exist? Nature Middle East 2013 (published online October 15, 2013).

[5] Abdul Hamid G, Tayeb MS, Bawazir AA. Breast cancer in southeast Republic of Yemen. East Mediterr Health J 2001;7;1012-16.

[6] El Saghir NS, Khalil MK, Eid T, El Kinge AR, Charafeddine M, Geara F, et al. Trends in epidemiology and management of breast cancer in developing Arab countries: a literature and registry analysis. Int J Surg 2007;5;225-33.

[7] Tarabeia J, Baron-Epel O, Barchana M, Liphshitz I, Ifrah A, Fishler Y, et al. A comparison of trends in incidence and mortality rates of breast cancer, incidence to mortality ratio and stage at diagnosis between Arab and Jewish women in Israel, 1979-2002. Eur J Cancer Prev 2007;16;36-42.

[8] Haddou Rahou B, El Rhazi K, Ouasmani F, Nejjari C, Bekkali R, Montazeri A, et al. Quality of life in Arab women with breast 
cancer: a review of the literature. Health Quality Life Outcomes $2016 ; 14 ; 64$.

[9] Mokdad AH, Forouzanfar MH, Daoud F, El Bcheraoui C, Moradi-Lakeh M, Khalil I, et al. Health in times of uncertainty in the eastern Mediterranean region, 1990-2013: a systematic analysis for the Global Burden of Disease Study 2013. Lancet Glob Health 2016;4;e704-13.

[10] GBD 2015 DALYs and HALE Collaborators. Global, regional, and national disability-adjusted life-years (DALYs) for 315 diseases and injuries and healthy life expectancy (HALE), 1990-2015: a systematic analysis for the Global Burden of Disease Study 2015. Lancet 2016;388;1603-58.

[11] GBD 2015 Eastern Mediterranean Region Cancer Collaborators, Fitzmaurice, C. Burden of cancer in the Eastern Mediterranean Region, 2005-2015: findings from the Global Burden of Disease 2015 Study. Int J Public Health 2017;63;151-64.

[12] GBD 2015 SDG Collaborators. Measuring the health-related Sustainable Development Goals in 188 countries: a baseline analysis from the Global Burden of Disease Study 2015. Lancet 2016;388;1813-50.

[13] The Lancet. GBD 2015: from big data to meaningful change. Lancet 2016;388; 1447 .

[14] Corbex M, Harford JB. Perspectives on breast cancer in Arab populations. Lancet Oncol 2013;14;e582.

[15] Najjar H, Easson A. Age at diagnosis of breast cancer in Arab nations. Int J Surg 2010;8;448-52.

[16] El Saghir NS, Shamseddine AI, Geara F, Bikhazi K, Rahal B, Salem ZMK, et al. Age distribution of breast cancer in Lebanon: increased percentages and age adjusted incidence rates of younger-aged groups at presentation. J Med Liban 2002;50;3-9.

[17] DeSantis C, Ma J, Bryan L, Jemal A. Breast cancer statistics, 2013. CA Cancer J Clin 2014;64;52-62.

[18] Chlebowski RT, Anderson GL. Changing concepts: menopausal hormone therapy and breast cancer. J Natl Cancer Inst $2012 ; 104 ; 517-27$.
[19] Helmrich SP, Shapiro S, Rosenberg L, Kaufman DW, Slone D, Bain C, et al. Risk factors for breast cancer. Am J Epidemiol $1983 ; 117 ; 35-45$.

[20] Ravichandran K, Al-Zahrani AS. Association of reproductive factors with the incidence of breast cancer in Gulf Cooperation Council countries. East Mediterr Health J 2009;15;612-21.

[21] GBD 2015 Obesity Collaborators. Health effects of overweight and obesity in 195 countries over 25 years. N Engl J Med 2017;377;13-27.

[22] Smigal C, Jemal A, Ward E, Cokkinides V, Smith R, Howe HL, et al. Trends in breast cancer by race and ethnicity: update 2006 . CA Cancer J Clin 2006;56;168-83.

[23] Nissan A, Spira RM, Hamburger T, Badrriyah M, Prus D, Cohen $\mathrm{T}$, et al. Clinical profile of breast cancer in Arab and Jewish women in the Jerusalem area. Am J Surg 2004;188;62-67.

[24] Donnelly TT, Al Khater A-H, Al-Bader SB, Al Kuwari MG, Al-Meer N, Malik M, et al. Beliefs and attitudes about breast cancer and screening practices among Arab women living in Qatar: a cross-sectional study. BMC Women's Health 2013;13;49.

[25] Vieira RA, Lourenço TS, Mauad EC, Moreira Filho VG, Peres SV, Silva TB, et al. Barriers related to non-adherence in a mammography breast-screening program during the implementation period in the interior of São Paulo State, Brazil. J Epidemiol Glob Health 2015;5;211-19.

[26] Mokdad AH, Jaber S, Aziz MIA, AlBuhairan F, AlGhaithi A, AlHamad NM, et al. The state of health in the Arab world, 19902010: an analysis of the burden of diseases, injuries, and risk factors. Lancet 2014;383;309-20.

[27] Rahim HFA, Sibai A, Khader Y, Hwalla N, Fadhil I, Alsiyabi H, et al. Non-communicable diseases in the Arab world. Lancet 2014;383;356-67.

[28] GBD 2015 Eastern Mediterranean Region Collaborators. Danger ahead: the burden of diseases, injuries, and risk factors in the Eastern Mediterranean Region, 1990-2015. Int J Public Health 2017;63;11-23. 